\title{
Musculoskeletal dysfunction in physical education teachers
}

\author{
Hélène Sandmark
}

\begin{abstract}
Objectives-To investigate the prevalence of musculoskeletal disorders, especially symptomatic osteoarthrosis (OA) of the knee and hip, health and lifestyle factors among physical education (PE) teachers, and whether they differed from the general population in these respects.

Methods-The study base was all Swedish men $(n=290)$ and women $(n=281)$ who graduated from the only training college for PE teachers in Sweden, between the years 1957 and 1965, and age matched referents, 255 men and 257 women, randomly selected from the Swedish population register. A postal questionnaire was sent to the subjects with questions on occupational history, participation in sports, musculoskeletal disorders, height, weight, smoking habits, and general health.

Results-The PE teachers had a higher prevalence ratio (PR) of symptomatic OA of the knee (men: 2.8 95\% confidence interval (95\% CI) 1.6 to 4.8 ; women: 3.2 $95 \%$ CI 1.8 to 5.5 ) and knee injury compared with the referents. The PE teachers reported more absence from work, and the women had had to change jobs more often because of knee disorders. The prevalence ratio for symptomatic OA of the hip was 2.7 (95\% CI 1.0 to 7.1$)$ for the female PE teachers. At the age of 25 the body mass index (BMI) of male PE teachers was higher than the male referents, but at an older age both the female and male PE teachers had lower BMI than the referents. The participation in sports activities was considerably higher in the group of PE teachers. They also smoked less, reported less serious diseases, and better health than the referents.

Conclusions-The PE teachers had a high risk of developing knee disorders, which entailed difficulties in continuing their work as PE teachers. They differed from the referents in lifestyle factors as they smoked less, were less overweight, and had a higher lifelong sports exposure. (Occup Environ Med 2000;57:673-677)
\end{abstract}

Keywords: body mass index; hip osteoarthrosis; knee osteoarthrosis

Several investigations have presented the prevalence of symptoms and radiographic osteoarthrosis (OA) of the knee in different occupational groups. Male dockers, ballet dancers, concrete workers, farmers, forestry workers, postmen, pipe fitters, and shipyard workers, and female cleaners and farmers showed an increased risk of OA of the knee in these studies. ${ }^{1-8}$ Female and male farmers, ballet dancers, fire fighters and food processing workers have been shown to have an increased risk of OA of the hip. ${ }^{6-11}$

According to the National Union of Teachers in Sweden, few physical education teachers are able to work in their profession until ordinary retirement due to various physical dysfunctions, mostly musculoskeletal. There is one earlier study on musculoskeletal disorders in this occupational group, where it was concluded that female PE teachers were not more prone to OA of the knee and hip than the general population. However, the cohort was created from registers of former female students, and only participation in sports and recreational activities was considered and not to what extent those teachers had been professionally active. ${ }^{12}$

The present study is the second part of two, on exposure and musculoskeletal disorders in physical education teachers. The first investigation aimed to measure exposure to physical load. ${ }^{13}$ The aim of this second part was to investigate the extent of musculoskeletal dysfunction, especially symptomatic OA of the knee and hip among PE teachers, and whether they differ from the general population in this respect. The purpose is also to show health and lifestyle factors in PE teachers.

Subjects and methods

The cohort included all Swedish men and women who graduated from Gymnastiska Centralinstitutet (GCI), the only training college for PE teachers in Sweden, between the years 1957 and 1965. Those who were born between the years 1925 and 1945, 290 men and 281 women, were included. At the time of this investigation the female teachers ranged between 53 and 65 years of age (median 57), and the male teachers between 54 and 72 (median 58). To identify the cohort the original register of students was identified, which is kept at the Swedish National Archives (Riksarkivet). The central population register was used to get the present address of each teacher, to get in touch with him or her for this investigation. To what extent the cohort was reached, and the participation rate is shown in table 1 . The PE teachers who had been working for less than 10 years in their profession $(n=60)$ were excluded from the analysis, as their occupational exposure as a PE teacher was considered too low.

Age matched referents in 5 year intervals, 255 men and 257 women, were randomly selected from the Swedish population register. 
Table 1 The cohort of physical education teachers who graduated in 1957-65, and the result of the identification of the cohort in 1996

\begin{tabular}{lcc}
\hline Cohort $(n=571)$ & $\begin{array}{l}\text { Men } \\
n=290\end{array}$ & $\begin{array}{l}\text { Women } \\
n=281\end{array}$ \\
\hline $\begin{array}{l}\text { Emigrated } \\
\text { Did not answer letter or telephone }\end{array}$ & 3 & 9 \\
Not in the central population & & 9 \\
$\quad$ register & 15 & 9 \\
Did not want to participate & 45 & 51 \\
Participation rate & $214(76 \%)$ & $202(72 \%)$ \\
Deceased & 10 & 2 \\
\hline
\end{tabular}

The participation rate for the male referents was $193(77 \%)$, and for the women $194(76 \%)$. Fifty three $(21 \%)$ of the male referents and 54 $(21 \%)$ of the female referents did not want to participate. Five of the men and three of the women referents could not be reached, and five women were too ill to be able to join the study.

A postal questionnaire was sent to the subjects with questions on sports participation during their youth and adult life, occupational history, musculoskeletal disorders and dysfunction, height, weight, smoking, and general health.

Information on participation in different kinds of sports activities was investigated from the age of 15 . The sports activities were reported for four periods of 10 year intervals and indexed. Those subjects who reported that they were physically active four times a week or more during one period got 20 points, and for reported sports activities one to three times a week 10 points were given. Eighty points was thus the maximum of sports exposure, and 50 points or more of lifelong sports exposure was considered high exposure.

Musculoskeletal pain, disorder, or dysfunction of different parts of the body now or in the past was asked for. For the hips and knees there

Table 2 The prevalence ratios (PRs) (95\% CIs) for self reported health, serious diseases, hypertension, and heart related symptoms among PE teachers versus the general population

\begin{tabular}{llllll}
\hline & \multicolumn{2}{l}{ Men } & & \multicolumn{2}{l}{ Women } \\
\cline { 2 - 3 } \cline { 5 - 6 } Variable & $P R$ & $95 \% C I$ & & $P R$ & $95 \% C I$ \\
\hline Good health & 1.3 & 1.1 to 1.5 & & 1.2 & 1.0 to 1.4 \\
Serious disease & 0.5 & 0.4 to 0.8 & & 0.7 & 0.5 to 0.9 \\
Hypertension & 1.0 & 1.0 to 1.1 & & 1.1 & 1.0 to 1.1 \\
Heart related symptoms & 1.1 & 1.0 to 1.1 & & 1.1 & 1.0 to 1.1 \\
\hline
\end{tabular}

Table 3 The prevalence ratios (PRs) (95\% CIs) for knee and hip injury, knee and hip osteoarthrosis $(O A)$, and knee and hip surgery among PE teachers compared with the referents

\begin{tabular}{llllll}
\hline & \multicolumn{2}{l}{ Men } & & \multicolumn{2}{l}{ Women } \\
\cline { 2 - 3 } \cline { 5 - 6 } Variable & $P R$ & $95 \% C I$ & & \\
\hline Knee injury & 2.1 & 1.6 to 2.8 & & 2.2 & $95 \% C I$ \\
Knee OA & 2.8 & 1.6 to 4.8 & & 3.2 & 1.6 to 3.0 \\
Knee OA adjusted for age & 2.7 & 1.6 to 4.6 & & 4.0 & 2.0 to 5.5 \\
Knee OA adjusted for BMI & 2.8 & 1.6 to 5.0 & & 4.5 & 2.3 to 8.9 \\
Knee OA adjusted for smoking & 2.4 & 1.3 to 4.4 & & 3.1 & 1.8 to 5.4 \\
Knee OA adjusted for sports & 2.6 & 1.4 to 5.0 & & 3.3 & 1.6 to 6.1 \\
Knee OA subjects with major & & & & \\
$\quad$ previous knee injury excluded & 2.1 & 0.5 to 8.5 & & 2.0 & 0.6 to 6.7 \\
Knee surgery & 3.4 & 2.3 to 5.2 & & 2.2 & 1.2 to 3.9 \\
Hip injury & 1.3 & 0.7 to 2.5 & & 1.3 & 0.7 to 2.5 \\
Hip OA & 0.9 & 0.5 to 2.0 & & 2.7 & 1.0 to 7.1 \\
Hip OA adjusted for age & 1.1 & 0.5 to 2.4 & & 3.0 & 1.1 to 8.1 \\
Hip OA adjusted for BMI & 1.0 & 0.5 to 2.2 & & 3.2 & 1.1 to 9.0 \\
Hip OA adjusted for smoking & 1.0 & 0.4 to 2.4 & & 2.8 & 1.0 to 8.1 \\
Hip OA adjusted for sports & 1.1 & 0.4 to 3.0 & & 5.5 & 1.2 to 25.2 \\
Hip surgery & 0.6 & 0.2 to 1.8 & & 1.4 & 0.4 to 5.2 \\
\hline
\end{tabular}

were questions on injury, diagnosis, and surgery. It was also asked if anyone had had sick leave, had changed jobs, or had claimed compensation due to musculoskeletal problems.

Body mass index (BMI), an indicator of overweight, was calculated as weight (kg) divided by height $\left(\mathrm{m}^{2}\right)$. The BMI of the referents was the basis for the classification of the BMI in three groups. The values in the highest quartile were considered as high BMI, and those in the lowest quartile as low BMI. The $50 \%$ in between were the medium BMI values. The BMI at the time when the subjects were 25 years of age, and at the time of the investigation, was calculated.

Cigarette smoking was calculated as packyears. One pack-year is the equivalent of 20 cigarettes/day for 1 year. The subjects were divided into three groups: never smokers, light smokers (1-14 pack-years), and smokers ( $\geqslant 15$ pack-years).

To obtain information about the general health of the subjects there were questions on self estimated health, and any past or present reported serious disease-such as cancer, diabetes, circulatory disease, metabolic disturbance, and others.

STATISTICAL ANALYSIS

The prevalence ratios (PRs) and 95\% confidence intervals (95\% CIs) for the variables investigated were calculated by comparing the cohort with the referents according to the Mantel-Haenszel method. ${ }^{14}$ Confounding from age, weight (expressed as BMI), smoking, and sports were controlled for also with the Mantel-Haenszel method. Due to the limited number of subjects the variables were analysed one at a time.

\section{Results}

The PE teachers reported fewer serious diseases and better general health than the referents (table 2).

The prevalence of symptomatic OA of the knee, earlier injury and surgery to the knee was higher in female and male PE teachers (table 3 ). The rate ratio for sick leave due to knee disorders was 1.7 (95\% CI 1.2 to 2.5 ) for the male, and 1.6 (95\% CI 1.1 to 2.5 ) for the female PE teachers. The female PE teachers also had a rate ratio of 3.7 (95\% CI 1.5 to 9.1 ) of having had to change work due to knee dysfunction, and among the men this increased rate ratio was 2.2 (95\% CI 0.9 to 5.6$)$. The PR for symptomatic OA of the hip was 2.7 (95\% CI 1.0 to 7.1 ) for the female PE teachers. Of the PE teachers $88 \%$ were still actively working in their profession at the age of 40 , and at the age of 50 the figure was $75 \%$. Of those who were 60 years of age at the time of the investigation, $19 \%$ were still working in this profession and seven women and nine men had taken early retirement. The remaining PE teachers had other jobs-such as specialist teachers, office workers, and principals.

The PE teachers who developed clinical OA of the knee or hip had been active in their profession between 10 and 39 years (median value 
31 years). Referents with OA of the knee and hip had been in various jobs, but mostly blue collar work. It was not possible to distinguish certain job titles entailing increased risk of OA as there was a wide variation.

Musculoskeletal disorders of the neck, shoulders, elbows, and back at any time in life were equally distributed among PE teachers and referents. However, there was a higher prevalence of disorders of the elbows among the female PE teachers, and of low back pain among both male and female PE teachers (table 4).

The PE teachers had been considerably more active in sports than the referents (table 5). Of the male PE teachers $73 \%(n=124)$, and of the women $40 \%(n=64)$ had at some time practised some sport at an elite level. Among the male referents $17 \%(n=32)$, and among the female referents $3 \%(n=5)$ had been at an elite level. Soccer, cross country skiing, downhill skiing, and jogging were the most frequent sports among both the male PE teachers and referents, and among the women gymnastics, jogging, cross country skiing, downhill skiing, and swimming.

Of the 34 male PE teachers with symptomatic OA of the knee, 31 reported previous high participation in sports (sports index $\geqslant 50$ points), and of the male referents four of nine with OA of the knee had had high participa-

Table 4 The prevalence ratios (PRs) (95\% CIs) for self reported musculoskeletal disorders in the neck, back, and upper limbs among PE teachers compared with the referents

\begin{tabular}{|c|c|c|c|c|}
\hline \multirow[b]{2}{*}{ Variable } & \multicolumn{2}{|c|}{ Men } & \multicolumn{2}{|c|}{ Women } \\
\hline & $P R$ & $95 \% C I$ & $P R$ & $95 \% C I$ \\
\hline Neck & 1.1 & 0.8 to 1.5 & 0.8 & 0.7 to 1.1 \\
\hline Shoulder & 1.3 & 0.9 to 1.7 & 0.8 & 0.6 to 1.0 \\
\hline Elbow & 1.0 & 0.7 to 1.6 & 1.6 & 1.1 to 2.3 \\
\hline Upper back & 0.9 & 0.5 to 1.6 & 1.0 & 0.7 to 1.4 \\
\hline Lower back & 1.1 & 1.0 to 2.1 & 1.2 & 1.0 to 1.4 \\
\hline
\end{tabular}

Table 5 Lifelong exposure (15-54 years of age) to sports among $P E$ teachers and referents $(n(\%))$

\begin{tabular}{lclll}
\hline $\begin{array}{l}\text { Sports } \\
\text { exposure in } \\
\text { index figures }\end{array}$ & $\begin{array}{l}\text { Male PE } \\
\text { teachers }\end{array}$ & $\begin{array}{l}\text { Male } \\
\text { referents }\end{array}$ & $\begin{array}{l}\text { Female PE } \\
\text { teachers }\end{array}$ & $\begin{array}{l}\text { Female } \\
\text { referents }\end{array}$ \\
\hline 0 & $1(0.6)$ & $52(32)$ & $1(0.6)$ & $68(43)$ \\
10 & $1(0.6)$ & $15(9)$ & $7(4)$ & $19(12)$ \\
20 & $3(2)$ & $22(14)$ & $7(4)$ & $23(15)$ \\
30 & $3(2)$ & $18(11)$ & $15(9)$ & $17(11)$ \\
40 & $49(31)$ & $33(20)$ & $56(35)$ & $23(15)$ \\
50 & $26(17)$ & $10(6)$ & $39(24)$ & $2(1)$ \\
60 & $27(17)$ & $3(2)$ & $11(7)$ & $2(1)$ \\
70 & $19(12)$ & $1(0.6)$ & $10(6)$ & $2(1)$ \\
80 & $27(17)$ & $8(5)$ & $16(10)$ & $3(2)$ \\
\hline
\end{tabular}

$\geqslant 50$ Index points is considered to be high exposure to sports.

Table 6 The prevalence ratios (PRs) (95\% CIs) for medium and high BMI (v low BMI) at the age of 25 and at the time for this investigation among PE teachers

\begin{tabular}{lll}
\hline & $25 y^{\star}$ & At present \\
\hline $\begin{array}{lll}\text { Men: } \\
\text { Medium BMI }\end{array}$ & $1.4(1.2$ to 1.6$)$ & $0.9(0.7$ to 1.1$)$ \\
$\quad$ High BMI & $1.6(1.2$ to 2.1$)$ & $0.4(0.2$ to 0.6$)$ \\
Women: & & \\
$\quad$ Medium BMI & $1.2(1.1$ to 1.4$)$ & $0.5(0.4$ to 0.6$)$ \\
High BMI & $1.2(0.9$ to 1.1$)$ & $0.1(0.1$ to 0.2$)$ \\
\hline
\end{tabular}

${ }^{\star} \mathrm{BMI}$ of men, low <20.7, medium 20.8-23.7, high $\geqslant 23.8$; women, low $<19.2$, medium $20.8-21.9$, high $\geqslant 22.0$.

†BMI of men, low $<23.9$, medium $24.0-27.8$, high $\geqslant 27.9$; women, low $<22.7$, medium $22.8-27.0$, high $\geqslant 27.1$. tion. Among the female PE teachers nine of 14 with OA of the knee reported high participation in sports, and of the three female referents with OA of the knee one had had high participation in sports.

At the time of the survey the BMI of PE teachers was considerably lower than the referents, but at the age of 25 the BMI of male PE teachers was higher than the male referents (table 6).

Among the PE teachers, $65 \%(n=109)$ of the men and $68 \%(n=110)$ of the women had never smoked, compared with $38 \%(n=66)$ of the male, and $55 \%(n=101)$ of the female referents. Among the male referents $35 \%(n=62)$ had 15 or more pack-years, and among the male PE teachers the figure was $7 \%(n=11)$. The figure for the female referents for 15 or more pack-years was $18 \%(n=33)$, and for female PE teachers $6 \%(n=10)$.

\section{Discussion}

The results of this study show evidence of more frequent injuries and symptomatic $\mathrm{OA}$ of the knees in both female and male PE teachers, and more symptomatic OA of the hip among the female PE teachers than the referents. The PE teachers were more often subjected to knee surgery. The group of PE teachers reported more absence from work, and had to change their work or work tasks more often because of knee disorders. Their sports participation was high, and they differed substantially from the referents in this respect. The BMI of male PE teachers was higher than the male referents at the age of 25. However, at an older age both men and women PE teachers had considerably lower BMI than the referents. The PE teachers smoked less, and reported less serious diseases than the referents.

At the time when the cohort attended the GCI in the late 1950s and early 1960s, it was the only training college in Sweden for PE teachers. As this study has a retrospective design the exposed cohort was traced through the central population register in Sweden. Out of the cohort of $571 \mathrm{PE}$ teachers, 24 were not in the register and could not be found. Some of them could recently have died, and some could have emigrated without informing the authorities. The subjects who reported that they had emigrated were few, and had moved out of Sweden for various reasons - such as marriage and work. Those subjects in the cohort and among the referents who did not want to participate gave various reasons for this when they were contacted. As the number of refusals was moderate, the potential bias from nonparticipation would be small.

In epidemiological studies the absence or presence of musculoskeletal disorders is often studied with reports of pain and symptoms from the subjects in answer to questionnaires. These reports can be overestimated or underestimated, and the possibility that the PE teachers reported differently from the referents cannot be disregarded. Both during work and sports practice the symptoms could be aggravated, and therefore the $\mathrm{PE}$ teachers might report musculoskeletal dysfunction differently. 
For the diagnosis of $\mathrm{OA}$ the reporting was based on a physician's opinion, and thus it was clinical OA that has been investigated in this study. The course of OA is different compared with, for example, low back pain, which often comes and goes. Osteoarthrosis is an ongoing process that includes structural changes, which are probably established at the time when the diagnosis is settled, and even a person with sedentary habits would go to a physician due to pain.

Some misclassification of exposure due to memory deficiencies is unavoidable, but to remember regular participation in a sport activity is not too difficult, even if it is rather a long time ago. It is probably more difficult to report the quantity of the past sports participation.

There are several drawbacks in using the general population as a comparison group in a cohort study such as this one. The PE teachers are most likely to be a selected group of people who, at least at the time of choosing their training and profession, were fit and healthy enough to manage a physically demanding profession, and had a lower morbidity compared with the general population. The healthy worker effect $^{1516}$ could bias the results, because subjects who are employed in certain occupations often constitute a group with lower risk of developing many diseases compared with the population as a whole, simply because the employed group must be healthy enough to work. The unemployed part of the population therefore has a higher risk of becoming ill. Thus, in a study that compares the morbidity experience of a certain occupational group with that of the total population, the healthy worker effect could lead to an underestimation of the relative morbidity in the exposed group. ${ }^{15}{ }^{16}$ If the healthy worker effect were a contributing factor in the present study, which is most likely, the PRs would be underestimated.

The effect of high participation in sports on OA of the hip and knee has not been finally concluded. In some studies it has been shown that running or jogging, which was common among both men and women in our study, at a non-elite level would not be a risk factor for OA of the knee, ${ }^{17-19}$ but a study carried out by Spector $e t a l^{20}$ showed an increased risk of OA of both the hip and knee among former middle aged female ex-elite athletes. It was concluded in this last study that long term weight bearing sports activities, such as jogging, squash, hockey, badminton, and aerobics, are correlated with these disorders. In two Swedish studies it was shown that high participation in all kinds of sports increased the risk of OA of the hip in men and women, and that track and field and racket sports were the most hazardous for men. ${ }^{21}{ }^{22}$ In a Swiss study of OA of the hip a positive association has been found with running, ${ }^{23}$ however, no such correlation could be found in a study on former Finnish elite runners. ${ }^{24}$

Soccer was the most common sports activity among men, both referents and PE teachers in the present study. Former soccer players, both with and without former injuries, have been found to have an increased risk of OA of both the hip and knee, and elite players ran the highest risk. ${ }^{18} 25-27$

In the present study the participation in sports and the occupational exposure of a PE teacher are both approximations for exposure to mechanical load on the legs. Results from earlier studies suggest that repetitive use of the knees for impact loading and heavy lifting in occupational work are associated with OA of the knee. ${ }^{28-30}$ The increased prevalence of OA of the hip in female PE teachers could be explained by a higher exposure to physical load compared with the male PE teachers, or anatomical differences between men and women, in the pelvis and the hip joint.

Occupational physical load can be compared with loads from sports, but it has not been possible to distinguish the impact from either exposure on the knee or OA of the hip in this study. It was shown that the PE teachers differed from the general population in leisure time participation in sports. The number of people in the present study did not allow calculations of stratum specific estimates. The PE teachers' high participation in sports and their occupational exposure were highly correlated. It could be concluded that these two exposures probably have a joint modification effect on the development of knee disorders. Investigations of the occupational exposure among PE teachers have been performed during whole workdays and it was shown that the exposure on the legs was considerable, as were the circulatory demands. High participation in lifting was found, especially when supporting the students in apparatus gymnastics, which produced the heaviest lifts. ${ }^{13}$ From earlier studies on risk factors it has been established that lifting is a risk factor for OA of the knee. ${ }^{730}$

Confounding may occur if a risk factor other than the studied exposure is unequally distributed between the cohort and the referentssuch as participation in sports. Other factors associated with OA or other musculoskeletal disorders, which could also be confounders, such as age, obesity, and smoking, were adjusted for in all the analyses. Due to few subjects in the study these potential confounding factors were adjusted for one at a time. None of these exposure variables were found to be confounders, not even when controlling for sports. However, a certain confounding could have appeared, as the confidence intervals were wide (table 3).

In conclusion the PE teachers had more knee disorders than the referents from the general population. They more often had to change work due to knee dysfunction, and only a fifth of them were still working in their profession at the age of 60 . They had a higher lifelong participation in sports, smoked less, were less overweight, and they had fewer serious diseases than the general population.

I thank Dr Eva Vingård, Professor Christer Hogstedt, Ms Ebba von Rosen, Mr Patrik Schéele, and Mr Staffan Hultgren for their kind and generous cooperation. 
1 Kellgren JH, Lawrence JS. Rheumatism in miners, part II: $x$ ray study. Br f Ind Med 1952;9:197-207.

Partridge REH, Duthie JJR Rheumatism in dockers and civil servants: a comparison of heavy manual and sedentary workers Ann Rheum Dis 1968:27:559-67.

3 Wickström G, Hänninen K, Mattsson T, et al. Knee degeneration in concrete reinforcement workers. $\mathrm{Br} \mathcal{F}$ Ind $\mathrm{Med}$ 1983;40:216-19.

4 Von Nauvald G. Untersuchungen zur Häufigkeit professioneller Kniegelenkserkrankungen bei älteren Rohrschlossern im Hochseeshiffbau. Beitr Orthop Traumatol 1986;33:124-8.

5 Lindberg H, Montgomery F. Heavy labour and the occurrence of gonarthrosis. Clin Orthop 1987;214:235-6.

6 Andersson S, Hessel T, Norén A, et al. Degenarative joint disease in ballet dancers. Clin Orthop 1989;238:233-6.

7 Vingård $\mathrm{E}$, Alfredson L, Goldie I, et al. Occupation and osteoarthrosis of the hip and knee. Int $\mathcal{f}$ Epidemiol 1991;20: 1025-31.

8 Sandmark H, Hogstedt C, Vingård E. Primary osteoarthrosis of the knee in men and women as a result of lifelong physical load from work. Scand $\mathcal{f}$ Work Environ Health 2000;26:20-5.

9 Typpo T. Osteoarthrosis of the hip: radiological findings and etiology. Ann Chir Gynaecol 1985;74:5-38.

10 Thelin A. Hip joint arthrosis: an occupational disorder among farmers. Am f Ind Med 1990;18:339-43.

11 Axmacher B, Lindberg H. Coxarthrosis in farmers. Clin Orthop 1993;287:82-6.

12 Eastmond CJ, Hudson A, Wright V. A radiological survey of the hips and knees in female specialist teachers of physical education. Scand $\mathcal{F}$ Rheumatol 1979;8:264-8.

13 Sandmark H, Wiktorin C, Hogstedt C, et al. Physical workload in physical education teachers. Appl Ergon 1999;30 $435-42$.

14 Mantel N, Haenszel W. Statistical aspects of analysis of data from retrospective studies of disease. $\mathcal{F}$ Natl Cancer Inst 1959;22:719-49.

15 McMichael AJ, Spirtas R, Kupper LL, et al. Solvent exposure and leukaemia among rubber workers. fournal of exposure and leukaemia among rubber workers. Fourn

16 Ostlin P. Negative health selection into physically light occupations. F Epidemiol Community Health 1988;42:152-6.
17 Klünder KB, Rud B, Hansen J. Osteoarthritis of the hip and knee joint in retired football players. Acta Orthop Scand 1980;51:925-7.

18 Sohn RS, Micheli LJ. The effect of running on the pathogenesis of osteoarthritis of the hips and knees. Clin Orthop 1985;198:106-9.

19 Lane NE, Bloch DA, Jones HH, et al. Running, osteoarthritis and bone density. $\mathcal{F} A M A 1986 ; 255: 1147-51$.

20 Spector TD, Harris PA, Hart DJ, et al. Risk of osteoarthritis associated with long term weightbearing sports: a radiologic survey of the hips and knees in female ex-athletes and population controls. Arthritis Rheum 1996;39:988-95.

21 Vingård E, Alfredsson L, Goldie I, et al. Sports and osteoarthrosis of the hip. An epidemiological study. Am 7 Sports Med 1993;21:195-200.

22 Vingård E, Alfredsson L, Malchau H. Osteoarthrosis of the hip in women and its relationship to physical load from sports activities. Am f Sports Med 1998;26:78-82.

23 Marti B, Knobloch M, Tschopp A, et al. Is excessive running predictive of degenerative hip disease? Controlled study of former elite athletes. BMf 1989;299:91-93.

24 Puranen J, Ala-Keltola L, Peltokallio P, et al. Running and primary osteoarthrosis of the hip. BMF 1975;285:424-5.

25 Lindberg H, Roos H, Gärdsell P. Prevalence of coxarthrosis in former soccer players. Acta Orthop Scand 1993;64:165-7

26 Roos H, Lindberg H, Gardnell P, et al. The prevalence of gonarthrosis and its relation to meniscectomy in former gonarthrosis and its relation to meniscectomy
soccer players. Am $\mathcal{F}$ Sports Med 1994;22:219-22.

27 Kujala UM, Kettunen J, Paananen H, et al. Knee osteoarthritis in former runners, soccer players, weight lifters and shooters. Arthritis Rheum 1995;38:539-56.

28 Anderson JJ, Felson DT, Factors associated with osteoarthritis of the knee in the first National Health and Nutrition Examination Survey (NHANES 1): evidence for an association with overweight, race and physical demands of work. Am 7 Epidemiol 1988;128:179-89.

29 Cooper C, McAlindon T, Coggon D, et al. Occupational activity and osteoarthrosis of the knee. Ann Rheum Dis 1994;53:90-3.

30 Felson DT, Hannan MT, Naimark A, et al. Occupational demands, knee bending, and knee osteoarthritis: results from the Framingham study. F Rheumatol 1991;18:1587-92. 